\title{
Neue Hürden für den therapeutischen Fortschritt
}

Der Artikel «Kosteneinsparungen dank Änderungen der Preisgestaltung von Originalpräparaten» in der SÄZ Nr. 37 hat zu zwei Stellungnahmen von Repräsentanten der pharmazeutischen Industrie geführt, die wir im Folgenden wiedergeben. Gerne hätten wir unserer Leserschaft an dieser Stelle auch eine Einschätzung des Beitrags von Behördenseite präsentiert. Swissmedic erklärte sich auf unsere Anfrage als für die Preisgestaltung nicht zuständig und verwies an das Bundesamt für Gesundheit BAG. Dieses wiederum bedauerte, die Vorschläge von Andreas Keusch nicht kommentieren zu können, da das BAG mit Beurteilungen von Vorschlägen zum Gesundheitswesen Schweiz generell zurückhaltend sei und seine primäre Aufgabe darin bestehe, die Aufträge des Bundesrats und des Parlaments umzusetzen.

Die Redaktion

Thomas B. Cueni

Generalsekretär Interpharma

Korrespondenz: Thomas Cueni Interpharma Petersgraben 35 CH-4051 Basel

thomas.cueni@interpharma.ch

\section{Das Nachsehen hätten die Patienten}

Was ist ein gerechter Preis für innovative Arzneimittel? An der Frage des «iustum pretium», mit der sich schon St. Augustin beschäftigte, sind schon viele gescheitert. In seinem Beitrag «Kosteneinsparungen» dank Änderung der Preisgestaltung von Originalpräparaten» stellt Andreas Keusch einige interessante Überlegungen an, doch sein Lösungsvorschlag - kein Innovationszuschlag für neue Medikamente - macht das System nicht effizienter: Im Gegenteil, er würde neue Hürden für therapeutischen Fortschritt schaffen. Das Nachsehen hätten die Patienten. Die Idee, neuen Medikamenten erst dann einen höheren Preis und zudem einen längeren Patentschutz zu geben, wenn ihr Mehrnutzen zweifelsfrei belegt ist, mag ja auf den ersten Blick bestechend sein. Wenn die Folge ist, dass Firmen Forschungsprojekte schon gar nicht in Angriff nehmen, weil das Risiko als zu hoch betrachtet wird, ist es schon weniger gut. Abgesehen davon, dass die von Keusch vorgeschlagene Preiserhöhung und Patentverlängerung zu einem späteren Zeitpunkt im heutigen politischen Umfeld wenig realistisch ist, sollten die Hürden für die Pharmaforschung ab- und nicht ausgebaut werden.

In der Schweiz wird aufgrund des wirtschaftlichen Erfolgs von Firmen wie Novartis, Roche oder Actelion leicht vergessen, wie risikoreich Pharmaforschung bereits heute ist. Tatsächlich dauert die Erforschung eines neuen Arzneimittels bis zu 15 Jahre, und rund 1,5 Milliarden Franken müssen bis zur Marktzulassung in ein neues Medikament investiert werden. Als Folge der immer höheren Anforderungen und der komplexeren Krankheitsgebiete, auf denen geforscht wird, ist die Produktivität der Forschung gesunken. Noch Mitte der 90er Jahre schaffte jedes fünfte Medikament, das an Patienten in klinischen Phase-I-Studien getestet wurde, die Marktzulassung, im Jahre 2000 war es nur noch jedes zwölfte Medikament. Und von 10 Medikamenten, welche die Zulassung schaffen, sind gemäss den Studien der Tufts University nur zwei wirklich rentabel. Das Verschwinden vieler einst renommierter Pharmafirmen ebenso wie die schwankende Bewertung von Pharma- und Biotechfirmen an den Börsen ist Ausdruck dieses Risikos.

Wenn Keusch kritisiert, dass «die aktuelle Politik zur Preisfestlegung eine Aufnahme in die SL anhand einer limitierten Datenlage» begünstige, beziehungsweise «Höchstpreise» für «durchschnittliche Qualität resp. Forschung» verlangt würden, liegt er falsch. Die zwei Kriterien für die Beurteilung der Wirtschaftlichkeit eines neuen Medikaments - Preis im Ausland und therapeutischer Quervergleich - werden in der Schweiz sehr konsequent angewandt. Tatsache ist, dass schon Frau Bundesrätin Dreifuss in den 90er Jahren erstaunt feststellen musste, dass die Schweiz für neue Medikamente oft tiefere Preise bezahlt als das vergleichbare Ausland, während für alte Medikamente und Generika in der Regel das Umgekehrte galt. Diese Erkenntnis war der Grund für die Einführung des sogenannten «Inno- 


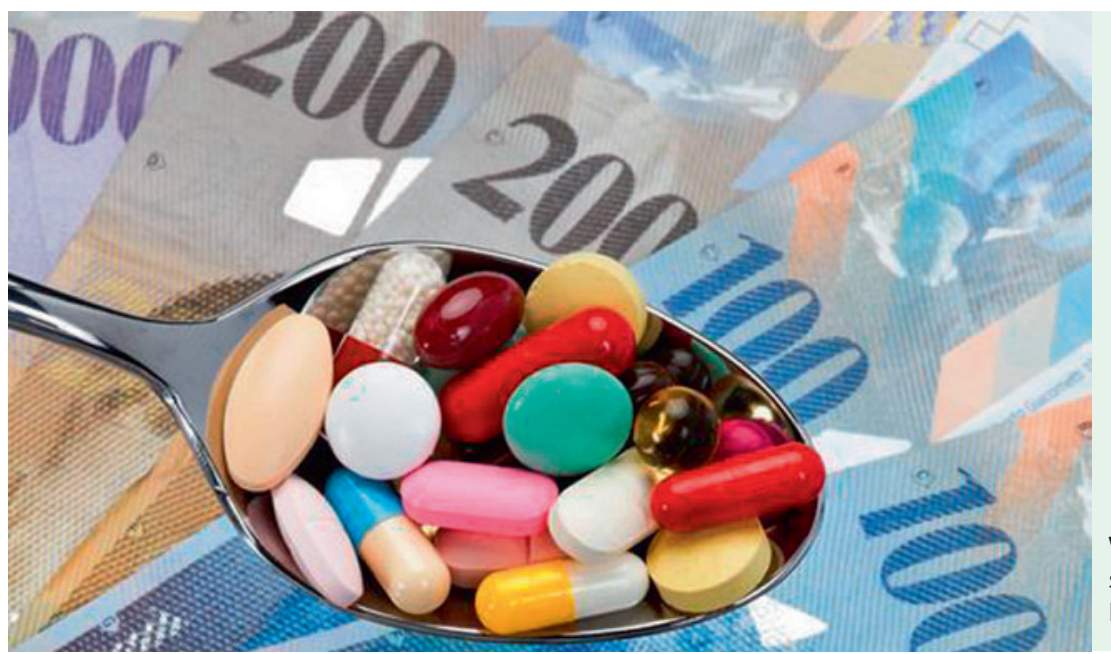

Wie viel darf die Forschung für innovative Medikamente kosten?

vationszuschlags» in den Verordnungen zum Krankenversicherungsgesetz. Die Umsetzung dieses Zuschlags blieb allerdings bis heute weitgehend Lippenbekenntnis. Im Vergleich zum Ausland ist man beim therapeutischen Quervergleich konservativ. An Beispielen dafür fehlt es nicht: Celebrex, der erste Cox-2-Hemmer, wurde in der Schweiz zum tiefsten Preis Europas eingeführt, und Peter Indra, Leiter der Abteilung Krankenversicherung im BAG, stellte schon mehrfach fest, dass die Schweiz bei innovativen Arzneimitteln in der Regel einen um 10-15 Prozent tieferen Preis hat als das vergleichbare Ausland. Dass die Schweiz bei neuen Produkten zurückhaltend ist, zeigt sich auch darin, dass Schweizer Patienten in jüngster Zeit länger auf die Kassenerstattung beispielsweise neuer Onkologika (u.a. Velcade, Avastin, Nexavar) warten mussten als Patienten in anderen europäischen Ländern. Diese Politik spiegelt sich auch in den Erhebungen von santésuisse, Preisüberwacher oder Interpharma wider, wo die Schweizer Preise neuer Produkte in aller Regel eher tiefer liegen als im vergleichbaren Ausland.

Zum Glück für den Werkplatz Schweiz sind die Kriterien für Investitionsentscheide in dem Pharmastandort multifaktoriell. Die Attraktivität für klinische Forschung in unserem Land wird durch die restriktive Praxis aber sicher nicht erhöht. Noch problematischer wäre es, wenn - wie Keusch de facto vorschlägt - ein neues Diabetesprodukt mit dem Preis von Metformin eingeführt werden müsste, ein neuer Lipidsenker nicht teurer sein dürfte als generisches Simvastatin, oder ein neues Antibiotikum sich am Preis generischen Amoxicillins orientieren müsste. Natürlich hat Keusch recht, wenn er schreibt, dass die Erstbeurteilung des NutzenRisiko-Profils neuer Produkte noch mit einer gewissen Unsicherheit behaftet ist. Korrekturen kann es allerdings in beiden Richtungen geben. Es gibt wohl Fälle wie Vioxx und Zelmac, bei denen ein Medikament aufgrund neuer klinischer Daten vom Markt genommen werden muss. Es ist aber selbst da nicht so, dass sich der wirtschaftliche Schaden für den Hersteller «dank der erzielten hohen Verkaufspreise und anfänglichen Markterfolgen» in «geringem Rahmen halten dürfte, da er den Innovationszuschlag zur Deckung der Kosten für Forschung und Entwicklung bereits bei der Markteinführung erhielt». Die Kosten späten Scheiterns sind immens und können wie bei der Abmagerungspille PhenPhen oder beim Rheumamittel Vioxx in die Milliarden gehen. Es gibt aber auch die gegenteiligen Fälle, in denen Arzneimittel die Erwartungen mehr als nur erfüllen. Dazu gehören etwa die neuen Biologika bei der rheumatoiden Arthritis oder Herceptin bei der adjuvanten Behandlung von HER-2-positivem Brustkrebs.

Zum Glück ist es auch nicht so, wie Keusch schreibt, dass «die Forschungsbestrebungen im Wesentlichen auf Neuentwicklungen fokussiert werden», weil die «nachweisliche Zweckmässigkeit nach Markteinführung als Belohnung für qualitativ hochstehende Forschung, Entwicklung und Marktüberwachung nicht honoriert» wird. «Risk Management Plans» stehen heute weit oben auf der Agenda von Zulassungsbehörden wie EMEA und FDA wie auch forschender Pharmafirmen. Es geht dabei sowohl um bessere Daten hinsichtlich Nutzen-Risiko-Profil wie auch um neue Anwendungen. So kann es durchaus vorkommen, dass ein neues Krebsmedikament wie Avastin die Erstzulassung aufgrund klinischer Daten mit wenigen hundert Patienten erhält, heute aber klinische Studien mit rund 40000 Patienten zu diesem Medikament laufen. Mit anderen Worten, manche grosse klinischen Studien beginnen erst nach Marktzulassung. Das Risiko für diese sehr kostenintensiven Studien liegt nicht bei der Allgemeinheit, sondern bei der Firma. Wenn sie Erfolg hat - wie bei Herceptin adjuvant - winkt ein Umsatzwachstum, wenn sich die Erwartungen (noch) nicht erfüllen - wie bei Avastin adjuvant -, reagiert die Börse rasch.

Der Umgang mit unsicherer Datenlage ist heute ein Thema vieler Konferenzen. Zum Glück hat die von Grossbritannien forcierte Idee, das Risiko, dass thera- 
peutische Hoffnungen nicht erfüllt werden, allein auf die Firmen zu verlagern, bis anhin wenig Nachahmer gefunden.

Der Regelfall ist eher das französische Modell, bei dem man einem neuen Arzneimittel aufgrund des medizinischen Bedürfnisses eine Bewährungschance gibt, die Erstbeurteilung aber korrigiert, wenn die Erwartungen nicht erfüllt werden. In diese Richtung sollten die auf Anfang Oktober in Kraft getretenen Verordnungsänderungen für die Wirtschaftlichkeitsprüfung von SL-Medikamenten gehen: Zum einen wird die routinemässige Überprüfung der Preise kassenpflichtiger Arzneimittel alle drei Jahre einen Einfluss auf die Entwicklung des schweizerischen Preisniveaus haben, zum andern wird man in diesem Zusammen- hang sicher auch eine Lösung dafür finden, wenn Kosten und Nutzen eines Medikaments aufgrund neuer klinischer Daten anders beurteilt werden müssen als bei der Aufnahme.

Wenn man therapeutischen Fortschritt will, braucht es auch in Zukunft ökonomische Anreize, nicht nur für den medizinischen Durchbruch, sondern auch um inkrementellen Fortschritt zu honorieren. Diesbezüglich verdient tatsächlich eine Idee von Andreas Keusch weitere Prüfung: die Belohnung für die Weiterentwicklung bestehender Produkte und die Erforschung neuer Indikationsgebiete bei Medikamenten, die nur noch über eine kurze Patentlaufzeit verfügen. Für solche Forschung fehlt in der Tat heute oft der ökonomische Anreiz.

\section{Hans Groth}

Mitglied der Geschäftsleitung Pfizer Schweiz
Korrespondenz:

Dr. med. Hans Groth

Pfizer AG

Schärenmoosstrasse 99

CH-8052 Zürich

\section{Plädoyer gegen ein gigantisches Innovationshindernis}

Andreas Keusch schlägt in seinem Beitrag vor, die endgültige Preisfestlegung von humanpharmazeutischen Innovationen erst nach erfolgtem Nachweis der wirtschaftlichen Überlegenheit in der Kosten-Nutzen-Analyse unter Alltagsbedingungen vorzunehmen, also erst einige Jahre nach der Prüfung von neuen Medikamenten gemäss den gesetzlich klar geregelten Kriterien der Wirksamkeit, Zweckmässigkeit und Wirtschaftlichkeit. Mit diesem Vorschlag wird impliziert, dass das heute in der Schweiz praktizierte Zulassungs- und Preisfestlegungsverfahren (= Aufnahme in die SL) keine umfassenden Informationen zur Beurteilung der Zweckmässigkeit eines neuen Arzneimittels voraussetzt.

Dem ist nicht so, und den Ausführungen muss daher widersprochen werden.

Pharmazeutische Entwicklungen können pro zugelassenem neuem Molekül bis zu einer Milliarde USD kosten. Der von den Behörden und den verantwortlichen Ethik-Kommissionen geforderte Entwicklungsaufwand, insbesondere in der späten klinischen Entwicklung, hat die Zielsetzung, ein neues Medikament gegenüber den besten verfügbaren Vergleichspräparaten zu charakterisieren. Ein derartiges Programm an mehreren tausend Patienten kostet bis zu 300 Millionen USD.

Die so gewonnene Datenlage reicht im Allgemeinen aus, den Stellenwert einer Innovation auch aus pharmakoökonomischer Perspektive einzuschätzen, also Aussagen zur «Zweckmässigkeit» zu machen. Somit kann man Fragen zu Wirksamkeit und Sicherheit gar nicht von solchen zum therapeutischen Wettbe- werbsumfeld trennen. Deshalb ist Zweckmässigkeit wie im Beitrag von Andreas Keusch behauptet - nicht zweitrangig, sondern von Anbeginn integraler Teil der Arzneimittelentwicklung und -zulassung. Wenn ein Entwicklungs-Dossier nicht zur Beantwortung genau der von Keusch angesprochenen Fragen ausreicht, verfehlt die Arzneimittelentwicklung ihre Aufgabe.

Die Forderung von Keusch zur Durchführung zusätzlicher von der Allgemeinheit zu finanzierender «Feldversuche» ist nicht nur unrealistisch - sie ist auch ein fragwürdiger Kostentreiber und zudem eine kaum erfüllbare Aufgabe. Beim Lesen dieses Beitrages kommt dem Leser unwillkürlich der Gedanke, dass es sich dabei um einen Argumentationsversuch handelt, eine (zusätzliche) Hürde aufzubauen, um Fortschritt zu erschweren. Eine solche Hürde würde Entwicklungsaufwand und -dauer für jedes innovative Medikament noch weiter erhöhen. Dies, obwohl wir über ausreichende Standards verfügen, die bei richtiger Anwendung die geforderten Informationen schon heute liefern.

Mit den geäusserten Vorschlägen wird unweigerlich ein gigantisches Innovationshindernis etabliert. Dies kann nicht im Interesse von Medizin und Patienten sein.

\section{Literatur \\ 1 Keusch A. Kosteneinsparungen dank Änderung der Preisgestaltung von Originalpräparaten. Schweiz Ärztezeitung. 2009;90(37):1436-9.}

